\title{
Der Cinderella-Effekt: Die schulische Allokation von Flüchtlingskindern aus Eritrea und anderen Krisenregionen
}

\author{
Achim Hättich, Andrea Lanfranchi \\ Interkantonale Hochschule für Heilpädagogik, Zürich
}

\begin{abstract}
Zusammenfassung: Das Flüchtlingsthema ist in den Medien und der öffentlichen Diskussion sehr aktuell und umstritten. Eine wichtige Frage hierbei ist, wie es den betroffenen Menschen geht, insbesondere den Kindern und Jugendlichen. In der vorliegenden Untersuchung wurde anhand der Daten von 1999 bis 2016 der Zürcher Bildungsstatistik analysiert, inwieweit diese Kinder und Jugendlichen eine nicht normale Schullaufbahn absolvieren. Es zeigte sich in fast allen Kategorien, dass Kinder aus Flüchtlingsländern häufiger Sondermaßnahmen in Anspruch nehmen und öfter Klassen repetieren oder zurückgestuft werden. Sie absolvieren mit hoher Wahrscheinlichkeit eine weniger anspruchsvolle Berufslehre. Die Allokation stellt ein großes Problem dar, da bei diesen Kindern oftmals keine klaren Zuweisungskriterien bestehen. Um den Leistungsstand der Flüchtlingskinder besser einschätzen zu können, braucht es Abklärungsverfahren.
\end{abstract}

Schlüsselbegriffe: Flüchtlingskinder, Migration, Allokation, Sonderschulung

\begin{abstract}
The Cinderella Effect: The Educational Allocation of Children from Eritrea and Other Refugee Countries

Summary: The issue of refugees is very topical and controversial in the media and in public debate. An important question is how it affects especially the children and adolescents. In the present study, based on data from 1999 to 2016, the Zurich education statistics were examined to what extent these children and adolescents complete an unusual school career. In almost all categories, children from refugee countries are more likely to be found in special classes and special schools, as well as repetition or downgrading are more often. Children from refugee countries are more likely to complete a less demanding apprenticeship. Allocation is a major problem, as these children often lack clear allocation criteria. In order to be able to better assess the performance level of refugee children, clarification procedures are required.
\end{abstract}

Keywords: Refugee children, migration, allocation, special schooling

\section{Flucht, Trauma und Schule}

Die Flüchtlingskrise in Europa ab 2015 ist eines der beherrschenden Themen unserer Zeit und bestimmt in vielen Ländern die politische Agenda. Im Oktober 2017 waren in der Schweiz 66'177 Asylsuchende registriert (Staatssekretariat für Migration, 2017). Die meisten Asyl- gesuche der letzten zehn Jahre stammen von Personen aus Eritrea (Januar 2007 bis Oktober 2017: 43'282), gefolgt von Afghanis$\tan \left(18^{\prime} 319\right)$, Syrien (17'908), Nigeria (15’063), Sri Lanka (11'156), Somalia (9'993) und Irak (9'987). Der Anstieg der Asylzahlen hat sich zwar deutlich abgeschwächt, dafür ist das Thema in der politischen Diskussion umso virulenter. 
Besonders herausfordernd ist die Situation geflüchteter Kinder und Jugendlicher, und dies vor allem für sie selber. Neben den mehr oder weniger starken Belastungen vor, während und nach der Flucht und der Ungewissheit über ihr Aufenthaltsrecht kommt für sie das Erfordernis hinzu, eine neue Sprache zu erlernen und sich in einen neuen Kulturraum einzugliedern. Das stellt die Schule vor schwierige Aufgaben, vorwiegend im Bereich der Unterstützung dieser Kinder, aber auch in Bezug auf ihre Zuteilung in altersentsprechende Schulklassen bzw. in einen geeigneten Schultyp.

Die Forschungslage zu Schulleistungen von Kindern aus krisengeschüttelten Ländern ist sehr dürftig. Eine nicht näher zu bestimmende Anzahl dieser Kinder war vor der Flucht nicht oder nie in der Schule, auch weil eine regelmäßige Beschulung in Flüchtlingscamps nur schwer organisierbar ist, wie die UHNCR (2017) schreibt: "According to our latest education report, 3.5 million refugee children did not attend school in 2016. Only 61 per cent of refugee children attend primary school, compared with a global average of 91 per cent. As refugee children age, the obstacles to education increase. Just 23 per cent of refugee adolescents are enrolled in secondary school, compared to 84 per cent globally. For tertiary education the situation is critical. Only one per cent of refugee youth attends university, compared to 36 per cent globally." Das Hauptproblem vieler dieser Kinder und Jugendlicher sind die traumatisierenden Erfahrungen aufgrund von Krieg, politischer oder religiöser Verfolgung ihrer Angehörigen oder schlicht Armut und Deprivation (Korittko, 2016). Die Prävalenz psychischer Beeinträchtigungen bei Kindern aus krisengeschüttelten Regionen ist sehr hoch. Attanayake et al. (2009) berichten in ihrer Metaanalyse, dass bei $47 \%$ der untersuchten Kinder posttraumatische Belastungsstörungen und bei $43 \%$ Depressionen diagnostiziert wurden. Betancourt et al. (2012) replizierten diese Befunde. Verschiedene Studien mit jüngeren
Kindern mit Traumatisierungen, aber ohne Kriegserfahrung, stellen negative Zusammenhänge zwischen Traumaerlebnissen und Schulleistungen fest (Goodman, Miller \& West-Olatunji, 2012; Romano, Babchishin, Marquis \& Fréchette, 2015; Jimenez, Wade, Lin, Morrow \& Reichman, 2016). Kinder, vor allem aber Jugendliche in der Adoleszenz, sind bezüglich Traumafolgestörungen besonders vulnerabel (Landolt, 2004; Murthy \& Lakshminarayana, 2006). Differenziert betrachtet, kann man gemäß Weine et al. (2013) bei traumatisierten afrikanischen jugendlichen Flüchtlingen verschiedene Anpassungstypen unterscheiden, die mit der Anwesenheit und Bildung ihrer Eltern sowie mit der Nationalität zusammenhängen. In einer der wenigen schulbezogenen Studien fanden Stermaca, Elgiea, Dunlapa \& Kellya (2010) bei Highschool-Absolventen mit Kriegserfahrungen, verglichen mit nicht kriegserfahrenen Migranten und kanadischen Schülerinnen und Schülern, jedoch keine Unterschiede.

Aus der Resilienzforschung (Lösel \& Bender, 2008; Rutter, 1985; Werner, 1989) geht hervor, dass nicht jedes Kind, das traumatische Situationen erlebt hat, Lern- und Verhaltensstörungen entwickelt. Resilienz im Sinne von Widerstandskraft ist die Aufrechterhaltung der biopsychosozialen Gesundheit trotz hoher Störungsrisiken, die Entwicklung von Kompetenz unter aktueller Belastung, die Fähigkeit, sich von Traumata zu erholen und sich trotz Stress erfolgreich in der Gesellschaft zu integrieren (Wustmann, 2005). Insbesondere bei Kindern aus Multiproblem-Milieus wie oft im Falle von psychosozial belasteten Migrationsfamilien können diese Aspekte fließend ineinander übergehen (Speck-Hamdan, 1999). Widerstandskräfte liegen sowohl in konstitutionellen und erworbenen Persönlichkeitsmerkmalen als auch in den Ressourcen der Familie und des Umfeldes. Zu den bedeutendsten förderlichen Merkmalen der Umwelt gehört bei Kindern und Jugendlichen die 
Schule. Lehrpersonen wirken im optimalen Fall als protektiver Faktor bei Kindern in psychosozialen Notlagen, sozusagen als strukturelle „zweite Heimat“. Wenn hingegen die Schule früh und stark selektioniert und separiert, dann ist sie kein Schutz, sondern ein weiteres Risiko (Lanfranchi, 2012).

Generell weist eine Vielzahl von Studien nach, dass die Zuweisung von Schülerinnen und Schülern (SuS) zu einer Klasse oder Schulform nicht vom tatsächlichen Leistungsstand abhängig ist, sondern von der sozialen Schicht, dem Migrationsstatus und der regionalen Steuerung im Bereich Educational Governance (Kronig, 2003; OECD, 2015; Wendt \& Schwippert, 2017). Bei SuS mit Migrationshintergrund muss davon ausgegangen werden, dass sie aufgrund verschiedener Mechanismen institutioneller Diskriminierung prinzipiell schlechtere Chancen in der Schullaufbahn haben (Kronig, Haeberlin \& Eckhart, 2000; Lanfranchi, 2007; Hättich, 2013).

Alle diese Studien gehen von bereits bestehenden Schullaufbahnen aus und betrachten den Lernerfolg am Ende einer bestimmten Zeitperiode. Bei Flüchtlingskindern wurden Fragen der Allokation bisher wenig berücksichtigt. Die ungleich verteilten Bildungschancen könnten auch darin begründet sein, dass Flüchtlingskinder von Anfang an falsch zugewiesen werden, da keine vergleichbare Einteilung stattfindet, wie es bei einem Umzug oder Schulwechsel der Fall ist. Grundsätzlich besteht bei erstmaligem Eintritt in eine Schule eines Schweizer Kantons die Möglichkeit, einer Regelklasse zugewiesen zu werden, und zwar entweder in der altersentsprechenden Form (Kindergarten, Primarschule, Sekundarschule, Lehre nach dem Eidgenössischen Fähigkeitszeugnis EFZ) oder selektiv in eine besondere (separierte) Schulform (Aufnahme- und Kleinklassen, Sonderschule, Zwischenlösung vor der Berufslehre, Lehre nach dem Eidgenössischen Berufsattest EBA). Nach dieser Zuwei- sung kann es zur Korrektur einer Fehleinstufung kommen, entweder in Form einer Repetition oder des Überspringens einer Klasse oder einer Auf- oder Abstufung in der dreistufigen Sekundarschule I. Für schulisch integrierte SuS mit sonderpädagogischem Bedarf sind ferner am Beispiel des Kantons Zürich die integrierte Sonderschulung in der Verantwortung der Regelschule (ISR) und diejenige in der Verantwortung der Sonderschule (ISS) zu nennen. Zu unterscheiden sind also grundsätzlich Separierung und Regelklasse. Als dritte Kategorie existiert die Korrektur, die innerhalb eines bestehenden Regelschulsystems stattfindet. Erfasst werden im Folgenden alle Maßnahmen und Schulformen, die nicht der sukzessiven, aufbauenden Abfolge der Regelschule entsprechen.

Dieser Prozess der Allokation aufgrund des Status als Flüchtlingskind in eine nicht reguläre und nicht dem tatsächlichen Leistungsstand entsprechende Schullaufbahn wird hier nach dem Grimm-Märchen „Cinderella-Effekt" genannt: „Die schlechten ins Kröpfchen, die guten ins Töpfchen." Dabei geht es zunächst einmal nur um den reinen Tatbestand der Allokation sowie der Auswirkungen auf die schulischen Leistungen und das Wohlbefinden; die dafür verantwortlichen Gründe spielen fürs Erste keine Rolle.

\section{$2 \quad$ Methode}

\subsection{Fragestellung}

Mit der vorliegenden Untersuchung wird auf die Abweichungen in der Schullaufbahn fokussiert und nicht auf den Regelfall. Dadurch soll ermittelt werden, wie Flüchtlingskinder ihre Laufbahn in der Schule absolvieren. Zum einen gibt es die Hypothese, dass Flüchtlingskinder öfters in Schulformen zu finden sind, die einer Separierung entsprechen. Zum anderen 
wird die Hypothese aufgestellt, dass es wegen des oftmals unklaren Ausgangszustandes von Flüchtlingskindern häufiger zu Korrekturen kommt. Es wird angenommen, dass es Differenzen zwischen verschiedenen Flüchtlingsnationen gibt, und auch, dass ein anderes Vorgehen der Zuweisung erfolgt, ob es nur relativ wenige Flüchtlingskinder gibt, wie in den 2000er Jahren, oder sehr viele, wie in den letzten Jahren.

Folgende Fragestellungen werden behandelt:

1. Wie häufig werden SuS aus Flüchtlingsfamilien separativen Schulformen zugeteilt? Gibt es Unterschiede, in welche separative Schulformen sie kommen?

2. Wie häufig finden Korrekturen der erstmaligen Einteilung statt? Gibt es Unterschiede zwischen den verschiedenen Formen der Korrekturen?

3. Gibt es Veränderungen über die Zeit, auch angesichts der sich verändernden Flüchtlingssituation?

4. Gibt es Unterschiede zwischen den Flüchtlingsländern? Unterscheiden sich SuS aus den Flüchtlingsländern von SuS aus anderen Ländern?

\subsection{Stichprobe und Messinstrument}

Wir stützen uns in dieser Analyse auf die Bildungsstatistik (BISTA) der Bildungsdirektion des Kantons Zürichs (2017). Über eine Webplattform müssen alle Schulen ihre Daten über alle SuS liefern, hauptsächlich schulische Maßnahmen sowie soziodemografische Indikatoren wie Geschlecht, Alter, Erstsprache und Nationalität. Der Kanton Zürich wird deshalb gewählt, weil er nicht nur der einwohnerstärkste Kanton der Schweiz ist, sondern auch seit 1999 die am besten dokumentierten, elektronisch erfassten amtlichen Daten schulischer Laufbahnen in der Schweiz aufweist. Der Stichtag für den Vorschulbereich, die obligatorische
Schulzeit und die Sek II (Mittelschule und Berufsbildung) ist jeweils der 15 . September. Mittlerweile besteht die Datenbank aus über 3.76 Millionen Datensätzen und umfasst 18 Jahre seit 1999. Daraus werden die fünf zahlenmäßig stärksten aktuellen Flüchtlingsländer in der Schweiz berücksichtigt, die auch für Deutschland und Österreich relevant sind: Eritrea, Afghanistan, Syrien, Somalia und Irak. Diese Länder werden mit dem Rest aller anderen Nationen verglichen. In der Regel werden Quoten erwähnt, d.h. die spezifische Maßnahme wird in Beziehung gesetzt zum Total der SuS des jeweiligen Landes.

Wohlbemerkt: Im Kanton Zürich sind wie in den anderen Kantonen der Schweiz alle Flüchtlingskinder vom ersten Tag ihrer Ankunft an schulpflichtig, da dies über die Wohnsitznahme definiert wird. Für Papierlose gilt das Gleiche. Als Faustregel gilt, dass sie ihrem Alter entsprechend eingestuft werden, wobei freilich nach dem Leistungsstand geschaut wird, was zu Auf- oder Abstufungen von Anfang an führen kann (Bildungsdirektion, 2015).

\section{Ergebnisse}

\subsection{Häufigkeiten und zeitliche Veränderungen}

Auf den Kanton Zürich bezogen zeigen alle Flüchtlingsländer einen Anstieg der Zahl an Kindern, der besonders bei Eritrea markant ausfällt. Die Anzahl schulpflichtiger Kinder aus Eritrea steigt kontinuierlich an und nimmt seit 2008 überproportional zu (s. Abb. 1), davor lag sie sogar unter den Zahlen anderer Flüchtlingsländer. Im Jahr 2016 gab es 187-mal mehr SuS eritreischer Herkunft als 1999 (wobei die Schülerschaft insgesamt um $13.6 \%$ zugenommen hat). Im Schuljahr 2016/17 besuchten 1309 Kinder aus Eritrea eine Zürcher Schule. Das ist von den Migrationsländern außerhalb Europas die höchste Zahl an SuS. 


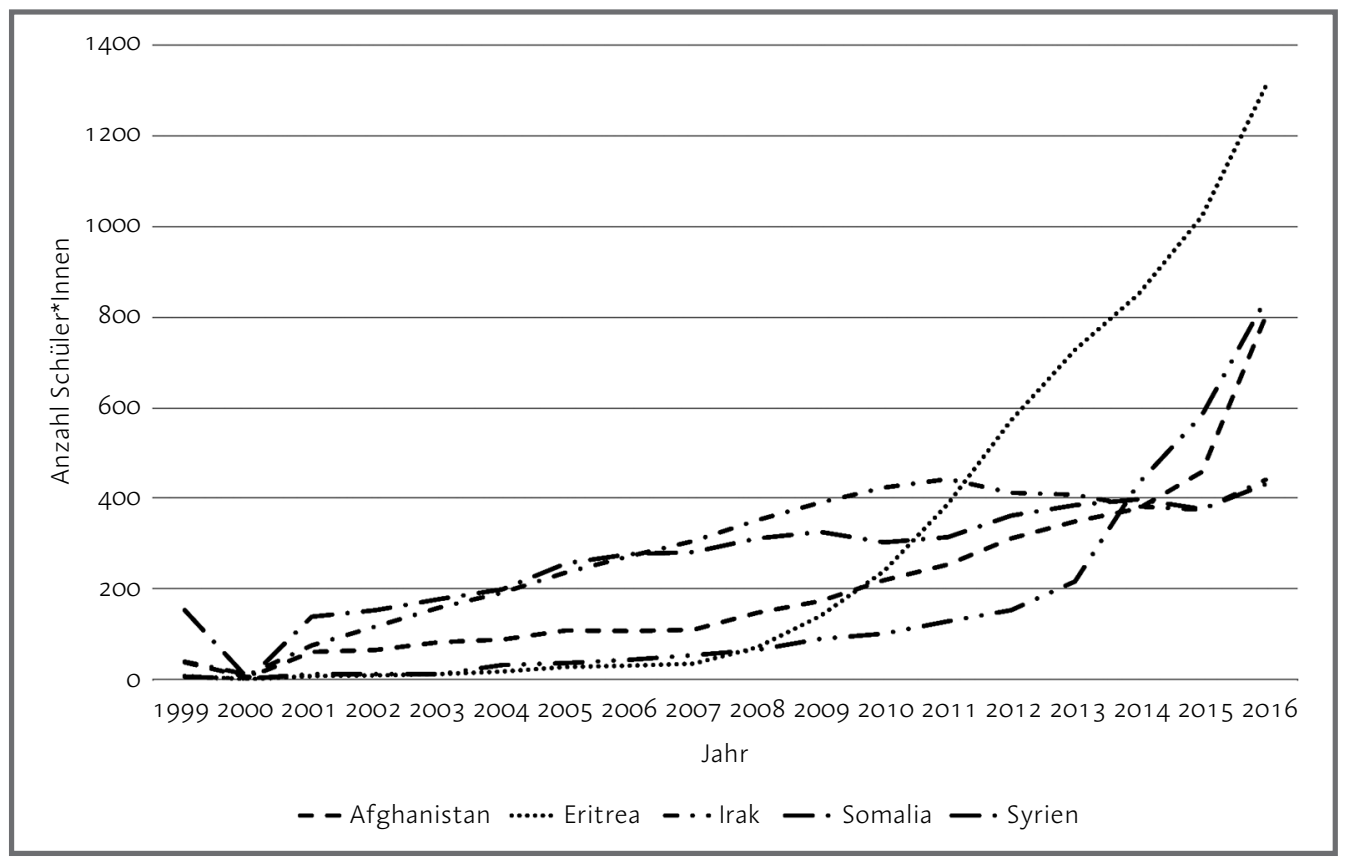

Abb. 1 Anzahl SuS ausgesuchter Flüchtlingsländer im Kanton Zürich 1999-2016. Diese und alle folgenden Abbildungen und Tabellen beruhen auf eigenen Berechnungen von Daten der Bildungsstatistik der Bildungsdirektion des Kantons Zürich 2017.

\subsection{Separierung}

\subsubsection{Aufnahme- und Einschulungsklasse}

Eine erste Möglichkeit für Kinder, die aus einem anderssprachigen Raum kommen und nicht direkt in einer regulären Klasse aufgenommen werden, ist der Besuch einer Aufnahmeklasse der Regelschule oder des Durchgangszentrums für Asylsuchende für maximal ein Jahr. Dort erwerben sie grundlegende Deutschkenntnisse und werden in allen Fächern altersgemäß unterrichtet. Eine zweite Möglichkeit - bei Schul-

Tab. 1 Anzahl und Quoten bei Aufnahme- und Einschulungsklassen nach Flüchtlingsland im Kanton Zürich kumuliert 1999-2016

\begin{tabular}{|l|c|c|c|c|c|c|}
\hline \multicolumn{2}{|l|}{ Art der Klasse } & Eritrea & Afghanistan & Irak & Somalia & Syrien \\
\hline \multirow{2}{*}{$\begin{array}{l}\text { Aufnahmeklasse } \\
\text { in Regelschule }\end{array}$} & Anzahl & 139 & 77 & 107 & 100 & 55 \\
\cline { 2 - 7 } & Quote & $3.0 \%$ & $1.9 \%$ & $1.6 \%$ & $1.5 \%$ & $2.4 \%$ \\
\hline $\begin{array}{l}\text { Aufnahmeklasse } \\
\text { Durchgangszentrum } \\
\text { Asylsuchende }\end{array}$ & Anzahl & 108 & 42 & 9 & 12 & 75 \\
\cline { 2 - 7 } & Quote & $2.4 \%$ & $1.1 \%$ & $0.1 \%$ & $0.2 \%$ & $3.2 \%$ \\
\hline Einschulungsklasse & Anzahl & 37 & 40 & 113 & 152 & 23 \\
\cline { 2 - 7 } & Quote & $0.8 \%$ & $1.0 \%$ & $1.7 \%$ & $2.3 \%$ & $1.0 \%$ \\
\hline
\end{tabular}


beginn - ist die Versetzung in eine Einschulungsklasse, die als sogenannte Besondere Klasse für Kinder geschaffen wurde, die zum Zeitpunkt des regulären Übertritts in die Primarstufe den Lernanforderungen in der 1. Klasse noch nicht gewachsen sind und für die ein weiterer Verbleib im Kindergarten nicht angezeigt ist. Sie schließt an die Kindergartenstufe an und dauert ein Jahr. Danach erfolgt der Übertritt in die 1. Regelklasse.

Kinder aus Eritrea mit fraglicher "Schulreife“ werden seltener in die Einschulungsklasse versetzt (s. Tab. 1), sondern viel eher für ein drittes Jahr in den Kindergarten zurückgestellt (resp. sie müssen das 2. Kindergartenjahr repetieren, was mit den unten stehenden Ausführungen betr. Repetitionen übereinstimmt). Tatsächlich hat Eritrea von allen berücksichtigten Nationen mit $6.1 \%$ den höchsten Anteil aller Kinder eines Landes auf Kindergartenstufe, die ein drittes Kindergartenjahr absolvieren.

\subsubsection{Sonderschule}

Ausgangsbasis für die folgenden Daten sind jene SuS, die in der BISTA-Datenbank unter der Rubrik „Heim- und Sonderschule“ gefasst sind. Bei allen Flüchtlingsländern bis auf Syrien steigt die Sonderschulquote in den drei Perioden $2000-2004,2005-2009$ und 2010 - 2016 kontinuierlich an (Abb. 2). In der Zeitperiode 2000 - 2004 lagen die Quoten generell niedriger (nur Somalia lag über der Quote der übrigen Länder), danach stiegen die Quoten kontinuierlich an. Die größeren Schwankungen bei den Flüchtlingsländern liegen zum Teil an der geringen Anzahl von Kindern aus diesen Ländern.

In Tabelle 2 finden sich die Daten zu den physiologisch determinierten Behinderungsarten, die bei Flüchtlingskindern bereits vor ihrer Einwanderung in die Schweiz bestanden haben oder Kriegsfolgen sind, wozu aber keine Daten existieren. Die Zahlen und Quoten sind

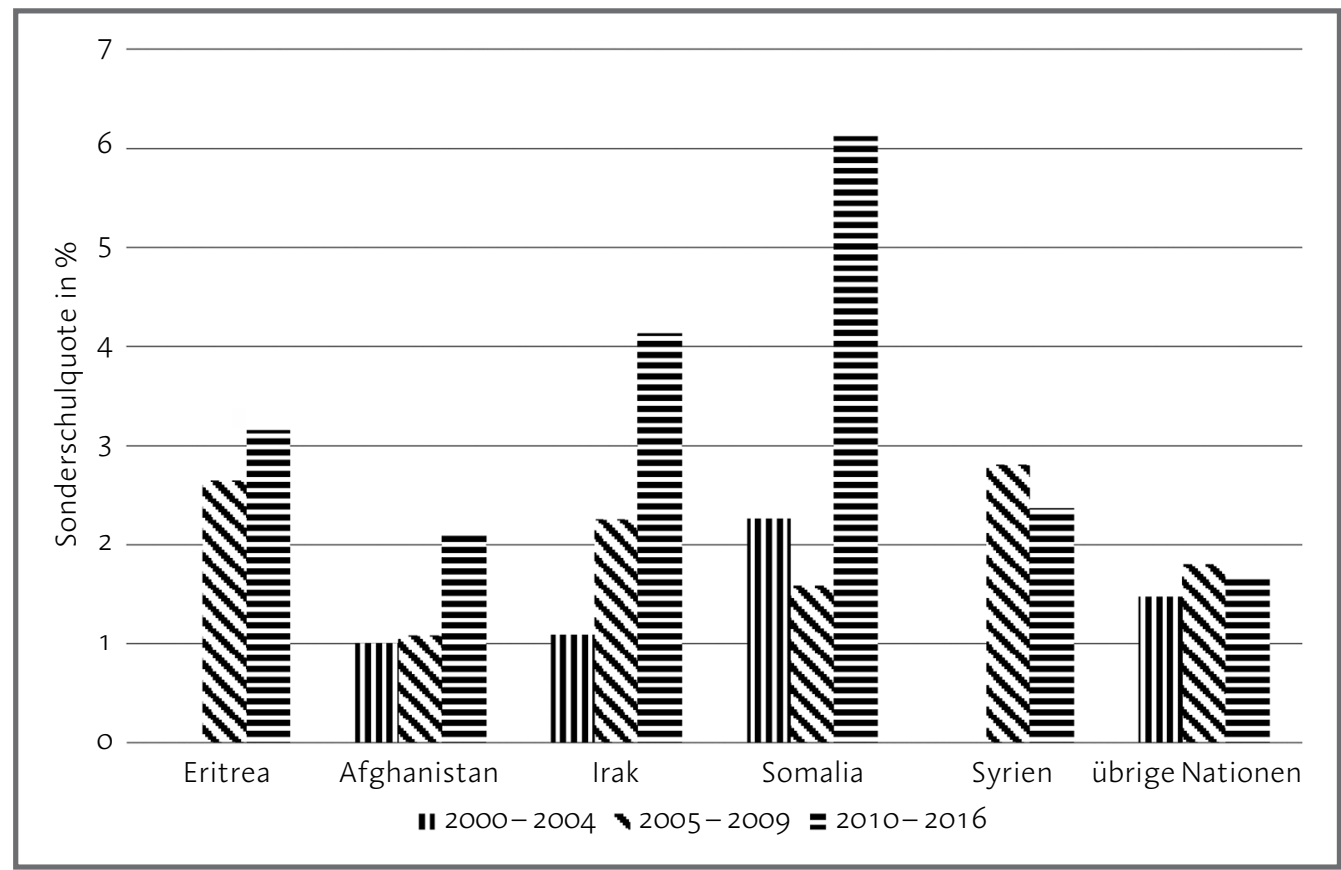

Abb. 2 Sonderschulquoten nach Flüchtlingsland im Kanton Zürich 2000-2016 
Tab. 2 Eher physiologisch bedingte Behinderungsarten nach Flüchtlingsland im Kanton Zürich $1999-2016$

\begin{tabular}{|l|l|c|c|c|c|c|c|}
\hline \multicolumn{2}{|l|}{ Art der Behinderung } & Eritrea & Afghanistan & Irak & Somalia & Syrien & Total \\
\hline \multirow{2}{*}{$\begin{array}{l}\text { Cerebrale } \\
\text { Lähmung }\end{array}$} & Anzahl & 4 & 3 & 1 & 0 & 1 & 9 \\
\cline { 2 - 8 } & $\%$ Quote & 0.07 & 0.08 & 0.02 & 0.00 & 0.04 & 0.03 \\
\hline \multirow{2}{*}{$\begin{array}{l}\text { Geistige } \\
\text { Behinderung }\end{array}$} & Anzahl & 71 & 34 & 60 & 87 & 24 & 276 \\
\cline { 2 - 8 } & $\%$ Quote & 1.30 & 0.91 & 1.19 & 1.80 & 0.85 & 0.19 \\
\hline \multirow{2}{*}{$\begin{array}{l}\text { Hör- } \\
\text { behinderung }\end{array}$} & Anzahl & 0 & 4 & 17 & 6 & 2 & 29 \\
\cline { 2 - 9 } & $\%$ Quote & 0.04 & 0.11 & 0.34 & 0.12 & 0.07 & 0.07 \\
\hline \multirow{2}{*}{$\begin{array}{l}\text { Körper- } \\
\text { behinderung }\end{array}$} & Anzahl & 0 & 0 & 8 & 2 & 2 & 12 \\
\cline { 2 - 9 } & $\%$ Quote & 0.00 & 0.00 & 0.16 & 0.04 & 0.07 & 0.04 \\
\hline \multirow{2}{*}{$\begin{array}{l}\text { Mehrfach- } \\
\text { behinderung }\end{array}$} & Anzahl & 11 & 3 & 14 & 3 & 6 & 37 \\
\cline { 2 - 9 } & $\%$ Quote & 0.20 & 0.08 & 0.28 & 0.06 & 0.21 & 0.10 \\
\hline \multirow{2}{*}{$\begin{array}{l}\text { Seh- } \\
\text { behinderung }\end{array}$} & Anzahl & 10 & 2 & 4 & 0 & 5 & 21 \\
\cline { 2 - 8 } & $\%$ Quote & 0.18 & 0.05 & 0.08 & 0.00 & 0.18 & 0.04 \\
\hline
\end{tabular}

Tab. 3 Eher psychologisch bedingte Behinderungsarten nach Flüchtlingsland im Kanton Zürich 1999-2016

\begin{tabular}{|l|l|c|c|c|c|c|c|}
\hline \multicolumn{2}{|c|}{ Art der Behinderung } & Eritrea & Afghanistan & Irak & Somalia & Syrien & Total \\
\hline $\begin{array}{l}\text { Lern- } \\
\text { behinderung }\end{array}$ & Anzahl & 16 & 0 & 3 & 9 & 5 & 33 \\
\cline { 2 - 8 } & $\%$ Quote & 0.29 & 0.00 & 0.06 & 0.19 & 0.18 & 0.15 \\
\hline $\begin{array}{l}\text { Psychische } \\
\text { Erkrankung }\end{array}$ & Anzahl & 0 & 0 & 3 & 7 & 0 & 10 \\
\cline { 2 - 8 } & $\%$ Quote & 0.00 & 0.00 & 0.06 & 0.14 & 0.00 & 0.05 \\
\hline $\begin{array}{l}\text { Sprach- } \\
\text { behinderung }\end{array}$ & Anzahl & 12 & 7 & 1 & 2 & 2 & 24 \\
\cline { 2 - 8 } & $\%$ Quote & 0.04 & 0.21 & 0.36 & 0.33 & 0.04 & 0.10 \\
\hline $\begin{array}{l}\text { Verhaltens- } \\
\text { behinderung } \\
\text { (Normal- } \\
\text { begabung) }\end{array}$ & Anzahl & 38 & 5 & 21 & 44 & 18 & 126 \\
\cline { 2 - 8 } & $\%$ Quote & 0.70 & 0.13 & 0.42 & 0.91 & 0.64 & 0.58 \\
\hline
\end{tabular}


jedoch gering, bis auf die Kategorie der kognitiven Beeinträchtigungen. Diese ist bei den Kindern aus allen Flüchtlingsländern höher als der kantonale Durchschnitt (0.55\%) und tritt bei Kindern aus Eritrea, Somalia und Irak gehäuft auf. Eine Mehrfachbehinderung ist bei Kindern aus dem Irak, Syrien und Eritrea häufiger als beim Durchschnitt (0.13\%). Eine Hörbehinderung tritt bei Kindern aus dem Irak fünfmal häufiger auf als beim Durchschnitt $(0.07 \%)$, während Sehbehinderung bei Kindern aus Eritrea und Syrien um das Achtfache öfter vorkommt als beim Durchschnitt (0.03\%).

Werden die mehr psychologisch bedingten, teilweise aber auch schulisch immanenten Behinderungen betrachtet (Tab. 3), liegen Lernbehinderungen bei Kindern von Eritrea deutlich über dem Durchschnitt von $0.18 \%$. Bei psychischen Erkrankungen und Verhaltensauffälligkeiten liegen Kinder aus Somalia deutlich über dem Durchschnitt (0.04\%, resp. 0.33\%). Bei Sprachbehinderungen weisen alle Flüchtlingsländer bis auf Syrien höhere Quoten auf (Durchschnitt: 0.20\%), markant sind hier die Unterschiede zwischen den Flüchtlingsländern.

\subsubsection{Zwischenlösung beim Übertritt Schule - Beruf}

Wer keine Lehrstelle findet oder keine weiterführende Schule besucht, für den oder die gibt es sogenannte Zwischenlösungen wie eine Vor- lehre oder das Berufsvorbereitungsjahr, die gewöhnlich von leistungsmäßig schwächeren Jugendlichen in Anspruch genommen werden (Tab. 4). Dabei gibt es spezielle Angebote für eingewanderte Jugendliche, wobei sich jedoch die Quoten unterscheiden. Das allgemein gehaltene Berufsvorbereitungsjahr „Sprache und Kultur" wird von Jugendlichen aus Eritrea und Somalia häufiger besucht, das Berufsvorbereitungsjahr "Integrationsorientiertes Angebot" häufiger von Jugendlichen aus Eritrea und Syrien.

\subsubsection{Eidgenössisches Berufsattest}

In der Schweiz gibt es zwei Arten von Berufslehren: die reguläre Berufsbildung EFZ (Eidgenössisches Fähigkeitszeugnis) mit einer Dauer von 3 bis 4 Jahren und das EBA (Eidgenössisches Berufsattest) mit einer Dauer von 2 Jahren, das für schulisch schwächere Jugendliche gedacht ist. Jugendliche aus Flüchtlingsländern absolvieren hochsignifikant häufiger ein EBA als ein EFZ $\left(\mathrm{Chi}^{2}=\right.$ 3786.21; $\mathrm{df}=5 ; \mathrm{p}=.000$ ). Besonders ist das bei Jugendlichen aus Eritrea der Fall: nahezu die Hälfte von ihnen absolviert ein EBA $(46.8 \%)$. Das ist im Vergleich $\mathrm{zu}$ anderen Flüchtlingsländern fast doppelt so hoch Irak $24.3 \%$, Somalia $24.1 \%$, Syrien $27.2 \%$, Afghanistan $27.4 \%$ - und zehnmal so hoch wie bei der Gruppe der restlichen Jugendlichen (4.8\%).

Tab. 4 Zwischenlösungen beim Übertritt Schule - Beruf nach Flüchtlingsland im Kanton Zürich kumuliert 1999-2016

\begin{tabular}{|l|c|c|c|c|c|c|}
\hline \multicolumn{2}{|l|}{ Art von Zwischenlösung } & Eritrea & Afghanistan & Irak & Somalia & Syrien \\
\hline \multirow{2}{*}{$\begin{array}{l}\text { Schulische } \\
\text { Zwischenlösung }\end{array}$} & Anzahl & 247 & 158 & 93 & 86 & 131 \\
\cline { 2 - 7 } & Quote & $0.05 \%$ & $0.04 \%$ & $0.02 \%$ & $0.02 \%$ & $0.05 \%$ \\
\hline \multirow{2}{*}{$\begin{array}{l}\text { Vorlehre/Berufs- } \\
\text { vorbereitungsjahr }\end{array}$} & Anzahl & 125 & 43 & 37 & 36 & 39 \\
\cline { 2 - 7 } & Quote & $0.02 \%$ & $0.01 \%$ & $0.01 \%$ & $0.01 \%$ & $0.01 \%$ \\
\hline
\end{tabular}




\subsection{Korrekturen}

\subsubsection{Repetition und Überspringen von Klassen}

Bei den Repetitionen weisen eritreische Kinder in der Schweiz die höchsten Werte auf (Abb. 3). Ein kontinuierlicher Anstieg zeigt sich auch bei den Kindern aus dem Irak, während bei den anderen Flüchtlingsländern der Trend nicht eindeutig ist und die Quote der übrigen Nationen stetig zurückgeht. Bei den Flüchtlingsländern schwanken die Quoten, was zum Teil an der geringen Anzahl Kinder liegt.

Andererseits überspringen im Vergleich zu den übrigen SuS durchschnittlich doppelt so viele somalische, syrische und irakische Kinder eine Klasse ( $0.6 \%$ vs. $0.3 \%$; keine Abb.). Eritreische und afghanische Kinder liegen mit $0.5 \%$ ebenfalls über dem Durchschnitt von $0.3 \%$. Dies dürfte mit der ursprünglichen Fehleinstufung zu tun haben.

\subsubsection{Auf- und Abstufungen in der Sekundarstufe I}

Beim Übertritt in die durchlässige Oberstufe (Sekundarstufe I) gibt es folgende Differenzierung:

Mittelschule (auch Gymnasium genannt): kognitiv anspruchsvollste Stufe

- Sekundarschule A (Sek A): kognitiv anspruchsvolle Stufe

- Sekundarschule B (Sek B): kognitiv weniger anspruchsvolle Stufe - Sekundarschule C (Sek C):

kognitiv am wenigsten anspruchsvolle Stufe

Je leistungsstärker der Sekundarstufentyp ist, desto seltener sind SuS aus Flüchtlingsländern darin vertreten. In der Sek A weisen eritreische Kinder mit $1.68 \%$ zusammen mit Kindern aus Somalia mit $1.61 \%$ den geringsten Anteil aller berücksichtigten Nationen auf (keine Abb.). Kinder aus Afghanistan haben mit $5.01 \%$ den

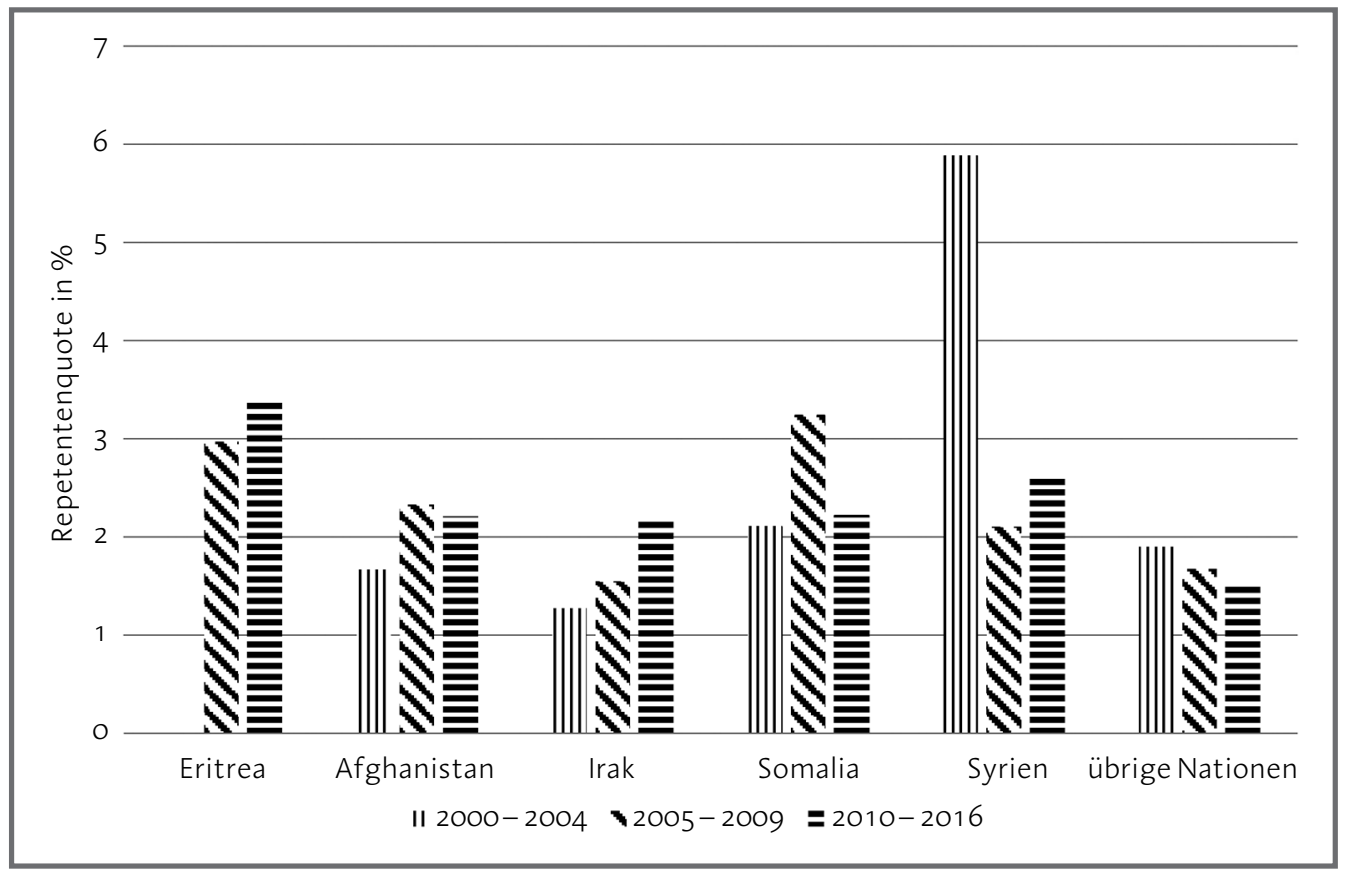

Abb. 3 Repetentenquote nach Flüchtlingsland im Kanton Zürich 2004-2016 


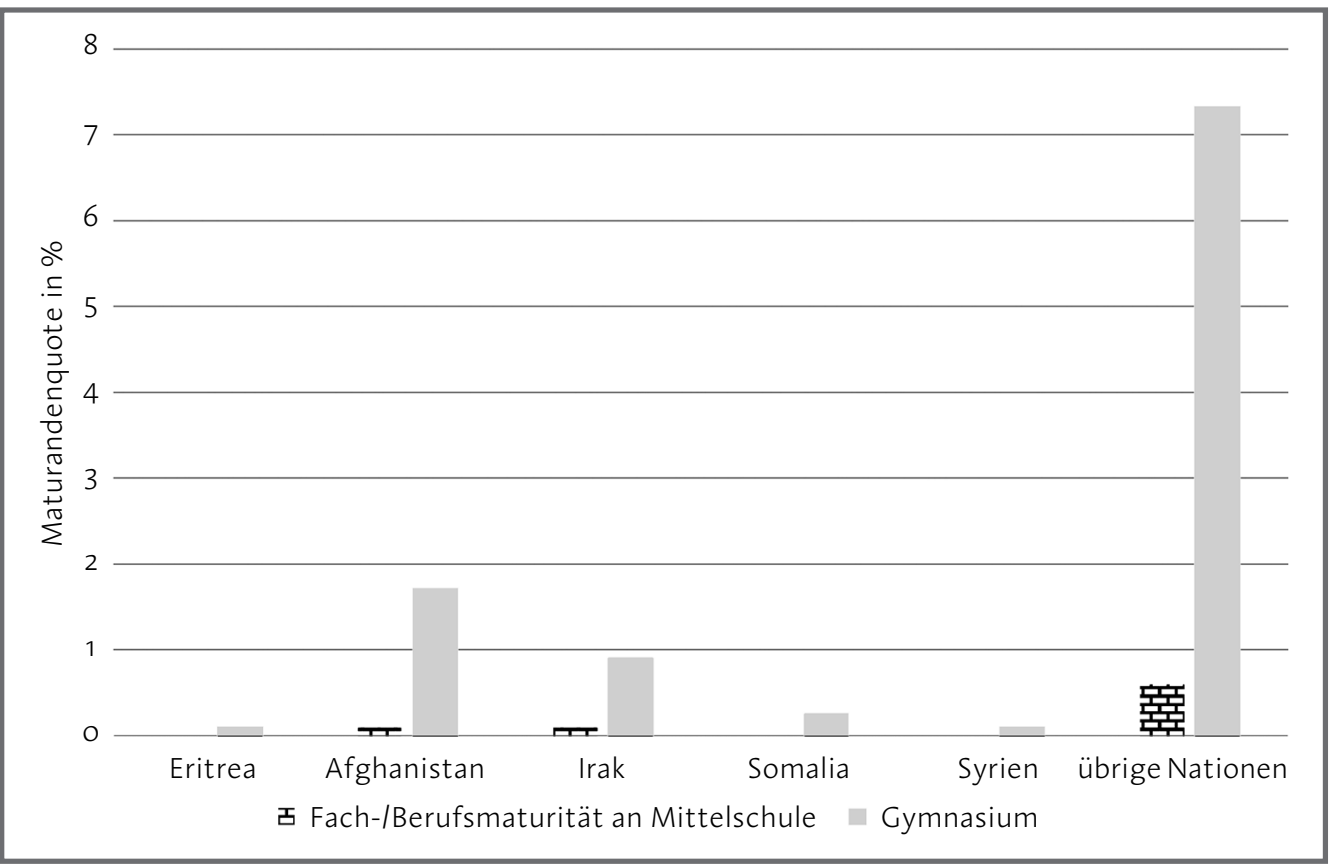

Abb.4 Anzahl SuS in Mittelschulen nach Flüchtlingsland im Kanton Zürich, kumuliert 1999-2016

höchsten Anteil, gefolgt von Kindern aus dem Irak mit $3.58 \%$ und aus Syrien mit $2.27 \%$, während die restlichen Nationen $8.30 \%$ aufweisen. In der Sekundarschule B liegen die Kinder aus allen Flüchtlingsländern über dem Durchschnitt. Bei der Sek C haben Kinder aus Syrien mit 3.59\% den höchsten Anteil, gefolgt von denjenigen aus Afghanistan mit 3.17\%, Somalia $2.98 \%$, Irak $2.88 \%$ und Eritrea $2.76 \%$, während von den restlichen Nationen nur $0.9 \%$ der Kinder in dieser Stufe sind. Alle Flüchtlingsländer sind signifikant seltener in Sek A und signifikant häufiger in Sek B und Sek C vertreten.

Bezogen auf die Mittelschule (inklusive Fachoder Berufsmaturität) liegt die Gesamtquote bei den 14- bis19-Jährigen für Jugendliche aus Afghanistan bei $5.1 \%$, gefolgt vom Irak 3.3\%, Somalia $0.8 \%$, Syrien $0.7 \%$ und Eritrea $0.1 \%$. Das sind im Vergleich zu den Quoten aller restlichen Nationen (18.0\%) auffallend tiefe
Anteile. Abbildung 4 zeigt, unabhängig vom Alter, die absoluten Häufigkeiten aller Kinder aus Flüchtlingsländern, die eine Mittelschule besuchen.

Als Indikator der Bildungsallokation fungiert, ob SuS in den leistungsmäßig unterschiedlichen Niveaus der Sekundarstufe I während ihrer Sekundarschullaufbahn in einen höheren Schultyp kommen (Aufstufung) oder in einen tieferen Schultyp (Abstufung). Von den übrigen Nationen werden durchschnittlich $0.61 \%$ abgestuft und $0.64 \%$ aufgestuft. Besser liegt Afghanistan mit $0.40 \%$ Abstufungen und $0.82 \%$ Aufstufungen. Während alle Flüchtlingsländer weniger Abstufungen haben als der Durchschnitt, haben sie ebenso weniger Aufstufungen: Irak (0.52\% Abstufungen, 0.49\% Aufstufungen), Syrien (0.39\%/0.14\%). Weniger Durchlässigkeit ist bei Somalia $(0.29 \% / 0.23 \%)$ und noch weniger bei Eritrea $(0.20 \% / 0.31 \%)$ festzustellen. 


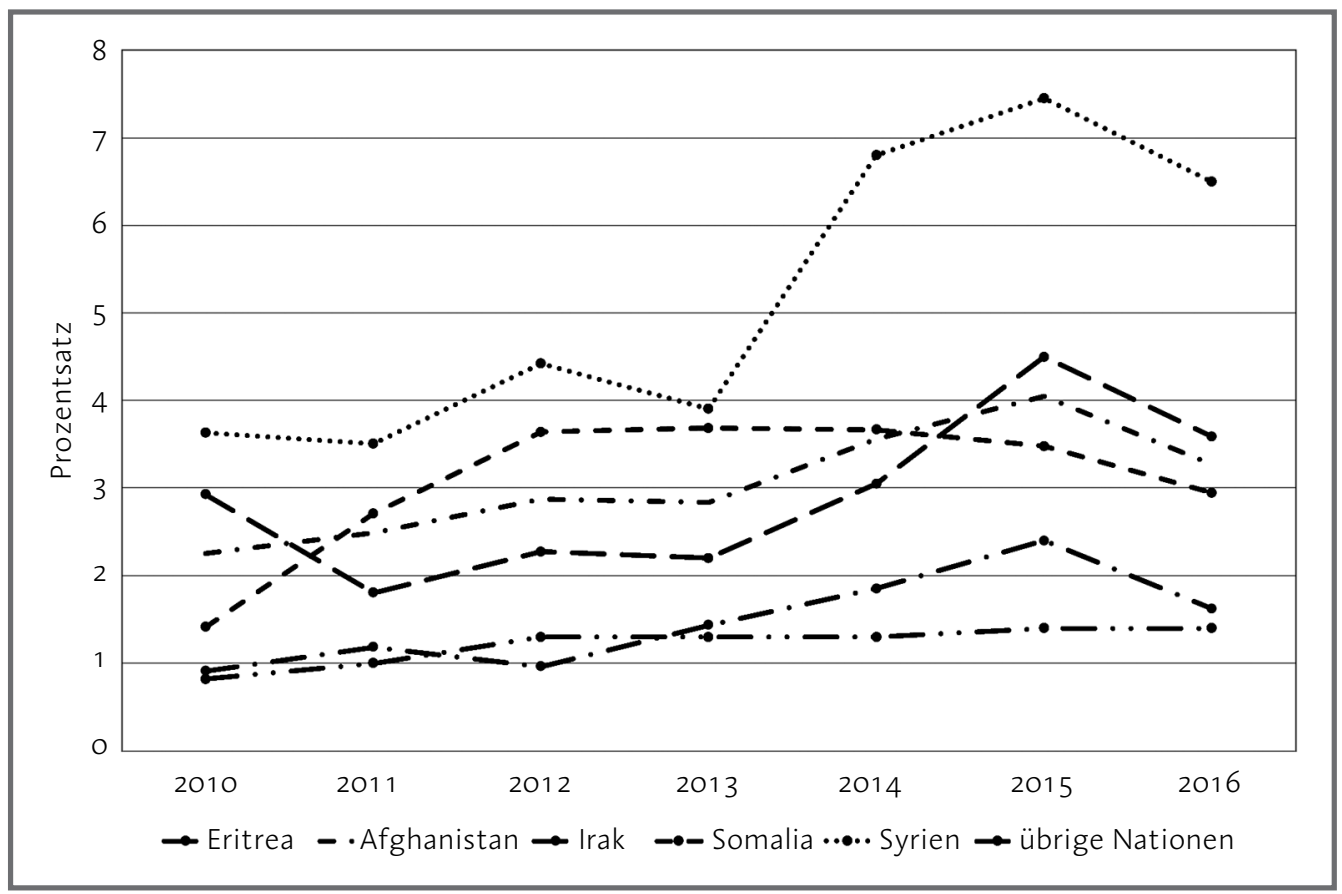

Abb. 5 Anteil Schüler und Schülerinnen mit integrierter Sonderschulung (ISR und ISS) nach Flüchtlingsland im Kanton Zürich 2010-2016

\subsubsection{Integrierte Sonderschulung}

Im Jahr 2010 erfolgte im Kanton Zürich die Einführung der ,integrierten Sonderschulung“ (sic!) an Regelschulen. Differenziert wird zwischen ISR (Integrierte Sonderschulung in der Verantwortung der Regelschule) und ISS (in der Verantwortung der Sonderschule): Kinder, die vorher in einer Sonderschule waren oder bei denen eine Sonderschule indiziert wäre, werden in die Regelschule integriert. Dies kann durchaus als Korrektur verstanden werden.

Bei allen Flüchtlingsländern nahm der Anteil an Kindern, die ISS oder ISR bekamen, über die Zeit bis $2015 \mathrm{zu}$, am stärksten bei SuS aus Syrien (Abb. 5). Alle Flüchtlingsnationen bis auf den Irak liegen deutlich über dem Anteil der übrigen Nationen. Im Jahr 2016 gab es bei allen Nationen einen Rückgang, der in dem Ausmaß in der Gesamtstichprobe nicht festgestellt wurde.

\subsubsection{Individuelle Lernziele}

Mit der Angabe „Individuelle Lernziele“ in der Integrativen Förderung wird erfasst, ob sich der Unterricht einer Schülerin oder eines Schülers danach ausrichtet, die Mindestziele des Regellehrplans der entsprechenden Schulstufe zu erreichen oder nicht. Ist das nicht der Fall, werden die Lernziele angepasst. Dies geschieht entweder dann, wenn in einem bis zwei Fächern nach individuellen, nicht dem Regellehrplan entsprechenden Zielsetzungen unterrichtet wird (Abb. 6: teilweise nach Lehrplan) oder wenn in drei oder mehr Fächern nach individuellen, nicht dem Regellehrplan entsprechenden Zielsetzungen unterrichtet wird (Abb. 6: mehrheitlich individuell).

Die syrischen und die eritreischen Kinder haben den höchsten Anteil jener, die in der integrativen Förderung nicht nach Regellehrplan unterrichtet werden (Abb. 6). Jedes siebte Kind 


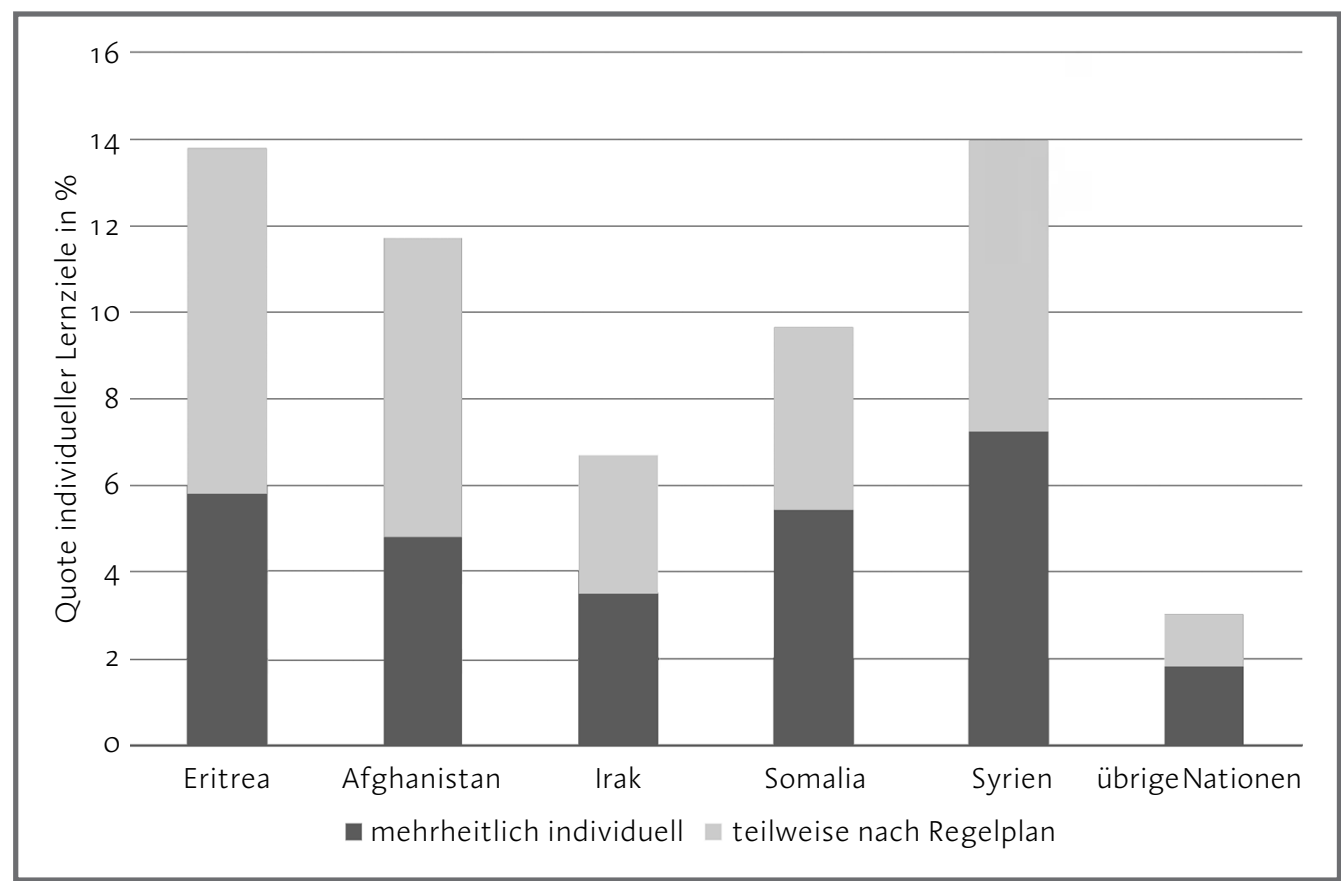

Abb. 6 Quote und Verteilung Individuelle Lernziele in der Integrativen Förderung nach Flüchtlingsland im Kanton Zürich 2010-2016

aus diesen beiden Nationen wird teilweise oder mehrheitlich individuell unterrichtet, womit diese Zahl fast um das Fünffache höher liegt als bei allen übrigen Nationen. Der Irak liegt hingegen nur doppelt so hoch.

\section{Schlussfolgerungen}

Die Anzahl von Kindern aus Flüchtlingsländern in der Schweiz - hier dokumentiert am Beispiel des Kantons Zürich - hat in den letzten Jahren stark zugenommen. Eine der Herausforderungen dabei ist, ihren Leistungsstand angemessen einzuschätzen, damit die entsprechende Klassenzuteilung korrekt vorgenommen werden kann. Fehleinteilungen aufgrund dürftiger Prognosen zeigen sich unter anderem in Form höherer Repetenten- und (seltener) Überspringerquoten, Ab- und (seltener) Aufstufungen in der Sekundarstufe I und im Rah- men der integrativen Förderung einer höheren Rate individueller Lernziele im Unterricht sowie Zuweisungen in besondere Klassen (Einschulungs- und Aufnahmeklassen), in den Typ der Berufslehre mit einfachsten Anforderungen (EBA) und Sonderschulung.

Die Hypothesen einer erhöhten Korrektur und einer höheren Separierung von Flüchtlingskindern können bestätigt werden. Zwischen den untersuchten Nationen gibt es Unterschiede, die aber über die verschiedenen Formen der Separierung und Korrektur keinen einheitlichen Trend aufweisen. Gleiches kann für zeitliche Veränderungen gesagt werden. Insgesamt zeigt sich in der Schullaufbahn von Flüchtlingskindern, entlang aller Maßnahmen, ein sehr komplexes Bild.

Die erstmalige Zuteilung eines Flüchtlingskindes erfolgt weitgehend aufgrund des Alters, das bei Aufnahme in eine Schweizer Schule 
deutlich höher liegt als bei Schweizer Kindern, die Leistungsfähigkeit wird dabei kaum berücksichtigt. Somit liegt es nahe, dass das Lernpotenzial von Flüchtlingskindern unter- oder überschätzt wird, was zu falschen Zuteilungen führt. Erschwerend kommt die Black-Box einer möglichen Traumatisierung vor oder während der Flucht bei ungefähr einem Drittel dieser Kinder hinzu (Lanfranchi \& Kohli, im Druck). Beides führt sowohl zu erhöhten Separierungsquoten als auch zu häufigeren Korrekturen. Um dem auf den Grund zu gehen, müssten längsschnittliche Analysen durchgeführt werden, in denen der Zeitpunkt von Separierung und Korrektur ermittelt wird. Jedenfalls weist dieser Sachverhalt darauf hin, dass ein Teil dieser SuS über beachtliche Bildungsvoraussetzungen verfügt, die bei der Einteilung in die jeweilige Schulklasse als solche nicht erkannt werden.

Aus Eritrea stammende Flüchtlingskinder weisen im Vergleich zu SuS aus anderen Flüchtlingsländern den höchsten Anteil an Separierungs- und Korrekturformen auf. Es gibt bedeutende Unterschiede zwischen diesen Formen, die allerdings aufgrund der geringen Häufigkeiten mit Vorsicht zu interpretieren sind. Die hohe Zuweisungsquote zum Typ der Berufslehre mit den einfachsten Anforderungen (EBA) bei eritreischen Jugendlichen liegt auch daran, dass viele von ihnen im Alter von über 16 Jahren, also nach der obligatorischen Schule, in die Schweiz einreisen. Was den zeitlichen Verlauf anbelangt, zeigten sich zu Beginn dieses Jahrhunderts noch relativ geringe Quoten von Separierungs- und Korrekturmaßnahmen, die jedoch bis 2010 stark zunahmen, danach war ein Rückgang zu beobachten. Beim Rückgang der Sonderschulungsquoten im Zeitraum von 2011 bis 2016 lassen sich die Trends der bildungspolitischen Entscheidungen im Bereich der schulischen Integration erkennen (Lanfranchi \& Steppacher, 2012). Nach den neuen Volksschulgesetzen und sonderpädagogischen Verord- nungen mit dem Primat der schulischen Integration (im Kanton Zürich 2005 resp. 2007, flächendeckende Umsetzung ab Schuljahr 2010/2011) nahmen die Zuweisungen in Sonderschulen ab. Dafür nahmen die Quoten der sogenannten integrierten Sonderschulung stark zu.

Relevant scheinen die Unterschiede zwischen den aufgrund physiologisch feststellbaren und somit relativ eindeutig diagnostizierbaren $\mathrm{Be}$ hinderungsarten wie Sinnes- und Mehrfachbehinderungen oder Cerebralparese und wenig eindeutig diagnostizierbaren und somit nicht immer vorurteilsfreien Kategorien wie Lern-, Verhaltens- und psychischen Behinderungen. Während bei den primär physiologisch bedingten Behinderungsformen die Quoten gering sind, liegen sie bei den vorwiegend psychologisch determinierten Kategorien höher. Dabei ist anzumerken, dass die absoluten Zahlen insgesamt klein sind.

Die schulische Integration von Kindern aus Flüchtlingsfamilien kommt nur schleppend voran. Hinsichtlich Schulerfolg handelt es sich um eine eher düstere Bilanz, was sich im Kanton Zürich in der aktuellen Bildungsstatistik widerspiegelt. Im Vergleich zu SuS aus anderen Nationen (inkl. Schweizer) sind Flüchtlingskinder deutlich übervertreten

- im Bereich der sogenannten integrierten Sonderschulung (Besuch von Regelklassen mit intensiver heilpädagogischer Unterstützung);

- Im Bereich der separierten Sonderschulung, vor allem im Falle kognitiver Beeinträchtigungen;

- in der Sek C (grundlegende Anforderungen), mit entsprechender Untervertretung in der Sek A (erweiterte Anforderungen) und verschwindend kleinen Mittelschulquoten;

v in der für leistungsmäßig schwächere Jugendliche vorgesehenen einfacheren Lehre des Typs Eidgenössisches Berufsattest (EBA). 
Eine solch ungleiche Verteilung von Bildungschancen bei SuS, die in Folge von Flucht und Einwanderung ihrer Familien als Asylsuchende erst seit wenigen Jahren in der Schweiz leben, ist erklärungsbedürftig. Wirft man einen Blick auf die Eritreer als die seit einigen Jahren größte Flüchtlingsgruppe, so findet man eine ganze Reihe von Belastungen, die obigen Befunden zugrunde liegen können.

In Eritrea dauert das Schulobligatorium acht Jahre, dennoch besucht ein beträchtlicher Teil der Kinder im schulpflichtigen Alter keinen Schulunterricht: Die Beschulungsquote liegt unter $50 \%$, Schulabbrüche und Repetitionen sind häufig, über $30 \%$ der Erwachsenen sind Analphabeten und des Lernens ungewohnt (EASO, 2015; UNESCO, 2012). Das letzte Schuljahr besuchen alle eritreischen Kinder mit 15 Jahren an einem zentralisierten Ort, wo sie für den Militärdienst vorbereitet werden (Eyer \& Schweizer, 2010). Die Repression des Landes und vor allem sich immer stärker ausbreitende Perspektivlosigkeit und Unplanbarkeit des eigenen Lebens haben sie während ihrer Kindheit durch die Leiden ihrer Familie erfahren. Von Kindern aus dem Irak oder jetzt aus Syrien unterscheiden sie sich dadurch, dass sie keine direkten kriegerischen Ereignisse erlebt haben. Deshalb sind Traumatisierungen bei eritreischen Kindern und Jugendlichen in der Zeit vor der Flucht gering. Hingegen können traumatisierende Erlebnisse während der Flucht auftreten (Burkhardt \& Lanfranchi, 2016). Zudem können die ersten Erfahrungen im Aufnahmeland für Kinder und Jugendliche aus krisengeschüttelten Regionen trotz sicherer Lage eine große Belastung darstellen, auch wenn sie nicht traumatisierend sind. Zum Beispiel in der ersten Zeit im Aufnahmezentrum angesichts des unklaren Asylstatus, nach der Verteilung auf die Kantone im Falle von Engpässen in den Wohnmöglichkeiten und eines Ausweichens auf unterirdische Zivilschutzanlagen und schließlich in der Schule im Falle von Aussonderung und somit erneuter Trennungserfahrungen.
Darüber hinaus gibt es weitere Gründe als Erklärung obiger Befunde, die nicht nur für die Flüchtlingskinder, sondern für alle Migrantenkinder gelten. Neben Schulschwierigkeiten aufgrund eingeschränkter Kompetenzen in der Lokalsprache und des sozialschichtbedingten geringeren Bildungsniveaus der Eltern (Gabel, Curcic, Powell, Khader \& Albee, 2009; Haenni Hoti, 2015) können Diskriminierungsmechanismen die überproportionale schulische Separation von Migrantenkindern erklären. Neben negativen Vorannahmen von Lehrpersonen (Lanfranchi, 2007) spielen systemische Prozesse eine Rolle: Sonderschulen als Organisation sind darauf angewiesen, ihre Plätze zu füllen. Wenn die Zahl der Sonderschüler und Sonderschülerinnen beispielsweise durch eine inklusive Praxis zurückgeht, nehmen Migranten deren Platz ein, wodurch die Regelschule eine schwierige Klientel loswird (Heckmann, 2008). Hättich (2013) wies nach, dass Migrantenkindern verstärkt das Etikett „kognitive Beeinträchtigung" zugewiesen wird, ohne dass eine Indikation dafür besteht. Vor ihm wiesen Kronig et al. (2000) empirisch nach, dass Migrantenkinder in ihren Fähigkeiten signifikant tiefer eingeschätzt werden als Schweizer Kinder mit identischen Leistungen, was eine negative Auswirkung auf ihre Lernentwicklung haben kann. Nach Kronig (2003) sind Erschwernisse im reibungslosen Ablauf des Unterrichtsalltags Ausgangspunkt von Stigmatisierungsprozessen: Die Versetzung von Migrantenkindern in besondere Klassen für Lernbehinderte korreliert nicht bzw. nur schwach mit den reellen sprachlichen und intellektuellen Leistungen dieser Kinder.

Sicher ist, dass ohne gezielte und individualisierte Förderung die üblichen Maßnahmen wie zum Beispiel sogenannte Einschulungsklassen, die Rückstellung in den Kindergarten und die Repetition der 1. Klasse bei der Einschulung wenig effektiv sind. Aus einem empirischen Vergleich ergeben sich jedenfalls keine Unterschiede in der Effektivität dieser Maßnahmen, die 
nachweislich zu keiner langfristigen Verbesserung des Schulerfolgs führen (Hermann \& NayCramer, 2002; Bless, Bonvin \& Schüpbach, 2004; Bayard, 2016; Törmänen \& Roebers, 2017).

Bei in die Schweiz geflüchteten eritreischen Kindern und Jugendlichen liegen besondere Belastungen vor, v. a. auch angesichts ihrer überdurchschnittlich häufig abwesenden Väter und der eher isolierten, teilweise überforderten alleinerziehenden Mütter (Burkhardt \& Lanfranchi, 2016). Präventive Investitionen im frühkindlichen Alter mit evidenzbasierten Förderprogrammen, wie in der Studie ZEPPELIN anhand des Hausbesuchsprogramms „PAT Mit Eltern Lernen“ evaluiert (Neuhauser et al., 2015), dürften bei in der Schweiz geborenen eritreischen Kindern ein Mittel der Wahl sein, um sich langfristig gerechten Bildungschancen anzunähern. Im Weiteren ist es wichtig, dass sich Lehrpersonen, Heilpädagoginnen und Heilpädagogen und Schulleitende bei Verdacht auf posttraumatische Belastungsstörungen sowie Anpassungsstörungen an die unterstützenden Dienste wenden, damit im Falle einer Indikation therapeutische Maßnahmen eingeleitet werden können (Lanfranchi, 2006; Lanfranchi \& Kohli, im Druck).

Auswirkungen schulimmanenter Benachteiligung zeigen sich darin, dass Kinder aus Flüchtlingsländern überdurchschnittlich häufig ausgesondert werden, was als "Cinderella-Effekt" bezeichnet werden kann. Ein „CinderellaEffekt" lässt sich auch in den Korrekturen der Schullaufbahn erkennen, die meist in eine negative Richtung gehen. Das alles ist zwar dadurch erklärbar, dass Flüchtlingskinder in vielen Fällen aufgrund ihrer Vorgeschichte und/oder aktueller Belastungen besondere Bildungsbedürfnisse aufweisen. Auf der anderen Seite sind Zuweisungsprozesse ohne prognostische Validität gerade bei Flüchtlingskindern besorgniserregend, weil sie nach schmerzhaften lebensbiografischen Einschnitten und in manchen Fällen sogar Traumatisierungen zu weiteren Brüchen führen, die zusätzlich traumatisierend wirken können. Aufgrund der vorliegenden Ergebnisse entsteht ein „Cinderella-Effekt" bei Flüchtlingskindern vor allem deshalb, weil nicht in hinreichender Art mit geeigneten Instrumenten und in genügender Dauer abgeklärt wird, welche Schulform und Schulstufe für ein bestimmtes Kind am besten geeignet ist. Zuteilungen erfolgen allein aufgrund des Status „Flüchtlingskind“ und vorwiegend auf das Alter gestützt, was allzu oft zu ungerechtfertigten Allokationen führt, da die Flüchtlingskinder, wenn sie in eine Schweizer Schule eintreten, älter sind als Schweizer Kinder bei Schuleintritt. Selbst eine exakte biologische (Nowotny, Eisenberg \& Mohnike, 2014) oder eine pädagogische Altersbestimmung ist in vielen Fällen nicht möglich, sodass hier die Gefahr einer falschen Allokation groß ist.

Bei den zugrunde liegenden Daten der Bildungsstatistik des Kantons Zürichs handelt es sich um eine Vollerhebung, die jährlich die Grundgesamtheit aller Schulkinder im Kanton Zürich individuell erfasst. Dennoch ist diese Datenbank nicht frei von Limitationen, welche die Aussagekraft einschränken. Angaben über den familiären Hintergrund fehlen fast vollständig, ebenso über soziale Schicht, Bildungsniveau und Lebenssituation der Eltern. Genauso sind Angaben über die psychische Befindlichkeit der Kinder nicht vorhanden, und bei Flüchtlingskindern fehlen Informationen über ihre schulische Vorgeschichte bis zur Anerkennung des Asylgesuchs. Ebenso wenig wird aus dieser Datenbank ersichtlich, ob das Kind in der Schweiz geboren wurde, infolge des ausländerrechtlich geregelten Familiennachzugs in die Schweiz eingewandert ist oder asylrechtliche Gründe für seinen Aufenthalt in der Schweiz zugrunde liegen. Im letzteren Fall fehlen Informationen über die Umstände, wie die Kinder in die Schweiz gelangt sind, und somit auch über mögliche Traumatisierungen. Angaben über die vor der Einreise in die Schweiz besuchten Klassen und Schulabschlüsse bei 
Jugendlichen fehlen gleichfalls. Nicht näher erfasst sind darüber hinaus Angaben zu pädagogisch-therapeutischen Maßnahmen wie Logopädie, Psychomotorik und Psychotherapie.

\section{Literatur}

Attanayake, V., McKay, R., Joffres, M., Singh, S., Burkle, F. \& Mills, E. (2009). Prevalence of mental disorders among children exposed to war: A systematic review of 7'920 children. Medicine, Conflict and Survival, 25 (1) 4-19. https:/l doi.org/10.1080/13623690802568913

Bayard, S. (2016). Bildungsverläufe während der obligatorischen Schulzeit. Zürich: Volksschulamt.

Betancourt, T.S., Newnham, E. A., Layne, C.M., Kim, S., Steinberg, M. A., Ellis, H. \& Birman, D. (2012). Trauma history and psychopathology in war affected refugee children referred for traumarelated health services in the United States. Journal of Traumatic Stress, 25(6), 682-69o. https://doi.org/10.1002/jts.21749

Bildungsdirektion des Kantons Zürich (2015). Flüchtlingskinder in der Volksschule. Informationen für Schulen und Gemeinden. Abgerufen am 3.12. 2017 von https:/ledudoc.ch/record/ 119094/files/fluechtlingskinder.pdf

Bildungsdirektion des Kantons Zürich (2017). Bildungsstatistik (Datensatz). Zürich: Bildungsdirektion des Kantons Zürich.

Bless, G., Bonvin, P. \& Schüpbach, M. (2004). Klassenwiederholung. Determinanten, Wirkungen und Konsequenzen. Bern: Haupt.

Burkhardt, S.C.A. \& Lanfranchi, A. (2016). Eritreische Flüchtlingskinder in der Schweiz. Schweizerische Zeitschrift für Heilpädagogik, $22(1)$, 20-26.

EASO (2015). COI Report. Eritrea Country Focus. Luxembourg: Publications Office of the European Union.

Eyer, R. \& Schweizer, R. (2010). Die somalische und die eritreische Diaspora in der Schweiz. Bern: Bundesamt für Migration.

Gabel, S. L., Curcic, S., Powell, J.J.W., Khader, K. \& Albee, L. (2009). Migration and ethnic group disproportionality in special education: an exploratory study. Disability \& Society, 24(5), 625-639. https://doi.org/10.1080/0968759090 3011063
Goodman, R.D., Miller, M. D. \& West-Olatunji, C. A. (2012). Traumatic stress, socioeconomic status, and academic achievement among primary school students. Psychological Trauma: Theory, Research, Practice, and Policy, 4(3), 252-259. https://doi.org/10.1037/aoo24912

Haenni Hoti, A. (Hrsg.) (2015). Equity - Diskriminierung und Chancengerechtigkeit im Bildungswesen. Migrationshintergrund und soziale Herkunft im Fokus. Bern: Schweizerische Konferenz der kantonalen Erziehungsdirektoren.

Hättich, A. (2013). Von draussen, nach draussen: Zur Separierung ausländischer Schülerinnen und Schüler. Schweizerische Zeitschrift für Heilpädagogik, 19(2), 28-34.

Heckmann, F. (2008). Education and Migration. Strategies for Integrating Migrant Children in European Schools and Societies. Brüssel: European Commission for Education and Culture. https://doi.org/10.2766/35262

Hermann, R. \& Nay-Cramer, D. (2003). Einschulungsklassen zur Prävention von Lernstörungen. In M. Brunstig, H. Keller \& J. Steppacher (Hrsg.), Teilleistungsschwächen - Prävention und Therapie. Luzern: Schweizerische Zentralstelle für Heilpädagogik, 131-145

Jimenez, M.E., Wade Jr. R., Lin, Y., Morrow, L.-M. \& Reichman, N.E. (2016). Adverse experiences in early childhood and kindergarten outcomes. Pediatrics, 137(2), 1-9. https://doi.org/10.1542/ peds.2015-1839

Korittko, A. (2016). Posttraumatische Belastungsstörungen bei Kindern und Jugendlichen. Störungen systemisch behandeln. Heidelberg: Carl Auer.

Kronig, W. (2003). Das Konstrukt des leistungsschwachen Immigrantenkindes. Zeitschrift für Erziehungswissenschaft, 6(1), 126-141. https:/l doi.org/10.1007/s11618-003-0008-3

Kronig, W., Haeberlin, U. \& Eckhart, M. (2000). Im migrantenkinder und schulische Selektion. Pädagogische Visionen, theoretische Erklärungen und empirische Untersuchungen zur Wirkung integrierender und separierender Schulformen in den Grundschuljahren. Bern: Haupt.

Landolt, M.A. (2004). Psychotraumatologie des Kindesalters. Göttingen: Hogrefe.

Lanfranchi, A. (2006). Kinder aus Kriegsgebieten in europäischen Einwanderungsländern. Trauma, Flucht, Schule und Therapie. systeme, 20(1), $82-102$ 
Lanfranchi, A. (2007). Sonderklassenversetzung und integrative Förderung: Denken und handeln Lehrpersonen kulturell neutral? Vierteljahresschrift für Heilpädagogik und ihre Nachbargebiete, 76(2), 128-141.

Lanfranchi, A. (2012). Resilienzförderung von Kindern bei Migration und Flucht. In R. Welter-Enderlin \& B. Hildenbrand (Hrsg.), Resilienz - Gedeihen trotz widriger Umstände, 119-138. 4. Aufl. Heidelberg: Carl-Auer-Systeme.

Lanfranchi, A. \& Steppacher, J. (Hrsg.) (2012). Schulische Integration gelingt. Gute Praxis wahrnehmen, Neues entwickeln. Bad Heilbrunn: Klinkhardt.

Lanfranchi, A. \& Kohli, C. (im Druck). Flüchtlingskinder - Integration dank Schule und Bildung. In T. Maier, N. Morina, M. Schick \& U. Schnyder (Hrsg.), Trauma - Flucht - Asyl: Ein praktisches Handbuch. Bern: Hogrefe.

Lösel, F. \& Bender, D. (2008). Von generellen Schutzfaktoren zu spezifischen protektiven Prozessen: Grundlagen und Ergebnisse der Resilienzforschung. In G. Opp \& M. Fingerle (Hrsg.), Was Kinder stärkt. Erziehung zwischen Risiko und Resilienz, 57-78. 3. Aufl. München: Reinhardt.

Murthy, R. S. \& Lakshminarayana, R. (2006). Mental health consequences of war: a brief review of research findings. World Psychiatry, 5(1), 25-30.

Neuhauser, A., Ramseier, E., Schaub, S., Burkhardt, S. C. A., Templer, F. \& Lanfranchi, A. (2015). Hard to reach families - a methodological approach for early detection, recruitment, and randomization in an intervention study. Mental Health and Prevention, 3(3), 79-88. https://doi.org/10. 1016/j.mhp.2015.07.002

Nowotny, T., Eisenberg, W. \& Mohnike, K. (2014). Unbegleitete minderjährige Flüchtlinge: Strittiges Alter - strittige Altersdiagnostik. Deutsches Ärzteblatt, 111 (18), 786-788.

OECD (2015).Immigrant Students at School: Easing the Journey towards Integration. Paris: OECD Publishing. http://dx.doi.org/10.1787/97892642 49509-en

Romano, E., Babchishin, L., Marquis, R. \& Fréchette, S. (2015). Childhood maltreatment and educational outcomes. Trauma, Violence, \&Abuse, 16(4), 418-437. https://doi.org/10.1177/15248 38014537908
Rutter, M. (1985). Resilience in the face of adversity: Protective factors and resistance to psychiatric disorder. British Journal of Psychiatry, 147(6), 598-611. https://doi.org/10.1192/bjp. 147.6.598

Slade, E. P. \& Wissow, L. S. (2007). The influence of childhood maltreatment on adolescents' academic performance. Economics of Education Review, 26(5), 604-614. https://doi.org/10.10 16/j.econedurev.2006.10.003

Speck-Hamdan, A. (1999). Risiko und Resilienz im Leben von Kindern ausländischer Familien. In: G. Opp, M. Fingerle \& A. Freytag (Hrsg.), Was Kinder stärkt. Erziehung zwischen Risiko und Resilienz, 221-228. München: Reinhardt.

Staatssekretariat für Migration (2017). Asylstatistik. Abgerufen am 3.12.2017 von https://www. sem.admin.ch/sem/de/home/publiservice/statistik/asylstatistik/archiv/2017/10.html

Stermaca, L., Elgiea, S., Dunlapa, H. \& Kellya, T. (2010). Educational experiences and achievements of war-zone immigrant students in Canada. Vulnerable Children and Youth Studies, 5(2), 97-107. https://doi.org/10.1080/1745012 0903440399

Törmänen, M.R.K. \& Roebers, C.M. (2017). Developmental outcomes of children in classes for special educational needs: results from a longitudinal study. Journal of Research in Special EducationalNeeds, online. https://doi.org/10.11 11/1471-3802.12395

UNESCO (2012). Global Education Digest $2012.0 p$ portunities Lost: The Impact of Grade Repetition and Early School Leaving. Montreal: UNESCO Institute for Statistics.

UNHCR (2017). Refugee Children are Left Behind. Abgerufen am 12.12.2017 von http://www. unhcr.org/education.html

Weine, S. M., Ware, N., Tugenberg, T., Hakizimana, L., Dahnweih, G., Currie, M., Wagner, M. \& Levin, E. (2013). Thriving, managing, and struggling: A mixed methods study of adolescent African refugees' psychosocial adjustment. Adolescent Psychiatry, 3(1), 72-81. https://doi.org/10.2174/ 2210676611303010013

Wendt, H. \& Schwippert, K. (2017). Lesekompetenzen von Schülerinnen und Schülern mit und ohne Migrationshintergrund. In: A. Hußmann, H. Wendt, W. Bos, A. Bremerich-Vos, D. Kasper, E. M. Lankes, N. McElvany, T. C. Stubbe \& R. Valtin (Hrsg.), IGLU 2016. Lesekompetenzen 
von Grundschulkindern in Deutschland im internationalen Vergleich, 219-234. Münster: Waxmann.

Werner, E.E. (1989). High-risk children in young adulthood: A longitudinal study from birth to 32 years. American Journal of Orthopsychiatry, $59(1), 72-81$.

Wustmann, C. (2005). Die Blickrichtung der neueren Resilienzforschung. Wie Kinder Lebensbelastungen bewältigen. Zeitschrift für Pädagogik, 51 (2), 192-206.

\section{Anschrift der Autoren}

Dr. Achim Hättich

Prof. Dr. Andrea Lanfranchi

Interkantonale Hochschule für Heilpädagogik

Schaffhauserstr. 239

Postfach 5850

$\mathrm{CH}-8050$ Zürich

E-Mail: achim.haettich@hfh.ch andrea.lanfranchi@hfh.ch 